\title{
Welches Verständnis haben Prosumer vom Urheberrecht im Web 2.0?
}

\author{
Eine qualitative empirische Untersuchung von jungen Erwachsenen
}

Thomas Rakebrand

\begin{abstract}
Zusammenfassung
Im Web 2.0 werden Internetnutzer/innen sowohl zu Konsumenten/-innen als auch zu Produzenten/-innen von urheberrechtlich geschützten Medieninhalten. Das OnlineHandeln dieser «Prosumer» (aus «producer» und «consumer») ist Mittelpunkt kontrovers geführter Debatten über das deutsche Urheberrecht: Während unter anderem Rechtswissenschaftler/innen eine Reformierung der Rechtsnormen zugunsten der von Nutzern/-innen erstellten Online-Inhalte ("User Generated Content») begrüssen, fordern vor allem die Urheber/innen und die Rechte verwertende Industrie den stärkeren Schutz des geistigen Eigentums und der Verwertungsrechte im Internet. Die Internetnutzer/innen, deren deviante Online-Nutzung im Fokus der Diskurse steht, wurden bislang kaum an diesen beteiligt und die wissenschaftliche Forschung hat sich unzureichend mit ihnen befasst.

In einer qualitativen empirischen Studie (Rakebrand 2014) wurde daher gefragt, welches Verständnis junge erwachsene Prosumer vom Urheberrecht im Web 2.0 haben was sie darüber wissen, welche persönlichen Erfahrungen sie damit gemacht haben, wie sie es bewerten und welche Beweggründe ihr urheberrechtsbezogenes Internethandeln hat. Die Untersuchung offenbart: Junge Erwachsene sehen sich ausserstande, das deutsche Urheberrecht in all seinen Facetten zu begreifen, es zu bewerten und sich an den öffentlichen Debatten darüber zu beteiligen. Um das Urheberrecht besser verstehen und mitgestalten zu können, fehlt ihnen ein «Leitfaden» zur Orientierung. Dieser Artikel fasst die zentralen Untersuchungsergebnisse zusammen und gibt Impulse für eine medienpädagogische Handlungspraxis.
\end{abstract}

\section{Prosumers and Their Understanding of the Copyright on the Web 2.0}

\begin{abstract}
On the web 2.0, Internet users are consumers as well as producers of media contents which are protected by the German Urheberrecht. The online activities of these so called "prosumers» play a central role in controversial debates about the German Urheberrecht: On the one hand, legal scholars, among others, prefer a reform of the legal norms to open them for online contents generated by users ("User Generated Content»). On the other hand, especially the creators of protectable work and the
\end{abstract}


industry that commercially exploits the work demand an increased protection of intellectual property and exploitation rights on the Internet. Prosumers hardly participate in discourses in which their deviant online use is the key topic and focus. Moreover, scientific research studied the prosumers insufficiently.

Within the scope of a qualitative empirical study (Rakebrand 2014) young adult prosumers were interviewed about their understanding of the German Urheberrecht on the web 2.0 - What do they know about it? Which personal experiences do they make? How do they evaluate it? And what are the reasons for their online use regarding the German Urheberrecht? The analysis reveals that young adults do not feel competent neither to understand the German Urheberrecht in all of its various aspects, nor to evaluate it and to participate in public debates. They lack a "guideline» for orientation to be able to understand and actively shape the German Urheberrecht. This article summarises key results of the study and provides impetus for media educational practice.

\section{Das Web 2.0 - ein rechtsfreier Raum?}

Im Jahr 2012 lehnte das EU-Parlament das besser unter dem Titel ACTA bekannt gewordene Anti-counterfeiting Trade Agreement ab. Gegner/innen des umstrittenen internationalen Urheberrechtsabkommens fürchteten um die Freiheit im Internet und warnten vor Einschränkungen der Rechte von Bürgern/-innen und Verbrauchern/-innen, während sich die Befürworter/innen um den Schutz des geistigen Eigentums, respektive um die Ausmasse des wirtschaftlichen Schadens durch Produkt- und Markenpiraterie sorgten. Massenhafte Proteste im Internet und europaweit auf der Strasse stoppten das Vorhaben vorzeitig. Eine ablehnende Haltung hinsichtlich der Anwendung bestimmter urheberrechtlicher Massnahmen im Internet offenbart sich auch am Beispiel des sozialen Online-Netzwerks Facebook. Dass vereinzelte Nutzer/innen der Plattform bereits abgemahnt wurden, weil die von Ihnen geteilten externen, urheberrechtlich geschützten Webinhalte als Miniaturvorschaubilder in ihre Profile eingebettet wurden, stösst auf ein drastisches Unverständnis junger erwachsener Prosumer (vgl. Rakebrand 2014).

Doch bedeutet dies auch, dass Internetnutzer/innen die Bestimmungen des deutschen Urheberrechts im Web 2.0 generell ablehnen und sich ein Internet ohne jedwede Regelungen wünschen? Ist daraus abzuleiten, dass sich hinsichtlich des geistigen Eigentums eine eigene Online-Moral etabliert habe, deren Credo es sei, alle Inhalte müssten für alle frei zu nutzen und damit auch zu reproduzieren sein? Darf gar konstatiert werden, das Urheberrechtsgesetz stecke in einer «digitale(n) Krise» (Gasser 2010, 76), wie die Befürchtungen in der Kreativindustrie es suggerieren? Um zu eruieren, wie es tatsächlich um das subjektive Verständnis der Prosumer hinsichtlich des Urheberrechts im Web 2.0 bestellt ist, wurde im Jahr 2013 eine qualitative empirische Untersuchung mit elf jungen Erwachsenen durchgeführt. Im 
vorliegenden Beitrag werden der Untersuchungsgegenstand, die bisherigen Forschungsbemühungen, das methodische Vorgehen sowie die zentralen Ergebnisse der Studie nachgezeichnet. Den Abschluss bildet ein Ausblick auf die medienpädagogische Praxis.

\section{Das Urheberrecht - Wandel und Debatte}

Was junge Erwachsene unter dem Urheberrecht verstehen und welchen Sinn sie den Regelungen im Web 2.0 aus ihrer subjektiven Sicht beimessen, ist kulturellhistorisch geprägt und unterliegt einem stetigen Wandel, da sich die konkrete Ausgestaltung des Urheberrechts seit seiner Entstehung kontinuierlich verändert. So war es bis zum Jahr 1850 als reines Vermögensrecht ausgestaltet (vgl. Höffner 2010, 3). Nicht die Urheberpersönlichkeit stand im Vordergrund, sondern die wirtschaftliche Auswertung der Nutzungsrechte. Zu den genuin vermögensrechtlichen Bestimmungen traten erst nach und nach Persönlichkeitsrechte hinzu - etwa das Recht, über die Veröffentlichung des eigenen Werkes zu bestimmen, sowie das Recht auf die Anerkennung der Urheberschaft (Höffner 2010, 52, 122). Das heute in der Bundesrepublik Deutschland geltende Urheberrechtsgesetz trat im Jahr 1965 in Kraft. In den letzten zehn Jahren wurde es rasant an die digitale (Online-) Nutzung von urheberrechtlich geschützten Werken angepasst (vgl. Kreutzer 2011, 4; Santangelo 2011, 36). Wesentliche Novellierungen waren der sogenannte Erste Korb (Bundesgesetzblatt 2003) sowie der Zweite Korb (Bundesgesetzblatt 2007). Bezogen auf diese Entwicklung konstatieren Rechtswissenschaftler/innen zum einen «Ausweitungen der urheberrechtlichen Ausschliesslichkeitsrechte zulasten der Nutzungsmöglichkeiten und -freiheiten auf Seiten der Nutzer sowie verschärfte Regelungen über die Durchsetzung von Urheberrechten, insbesondere im OnlineBereich» (Kreutzer 2011, 4), zum anderen eine «Tendenz zu einer flexibleren Handhabung urheberrechtlicher Schranken im Informationszeitalter» (Bauer 2011, 184). Damit einher gehen öffentlich geführte Debatten, die sich in den letzten Jahren verstärkt auf die Berechtigung der bisherigen urheberrechtlichen Bestimmungen im digitalen Zeitalter der Medien beziehen (vgl. hierzu Groß 2009, 203). In der Literatur werden mit Blick auf die relevanten Akteure/-innen dieser Debatten, etwa bei Bauer (2011), die prosumierenden Bevölkerungsmitglieder als Werknutzer/innen und die hiervon betroffenen Urheber/innen und Verwerter/innen als Rechteinhaber/innen fokussiert (vgl. Bauer 2011, 380). Diese Sichtweise auf eine «tripolare Interessenlage» (Lauber-Rönsberg 2012) greift jedoch erstens zu kurz, da an den Debatten - auch indirekt - eine Vielzahl weiterer Akteure/-innen beteiligt sind: wie die Gesetzgebung und Rechtsprechung, die Wissenschaft sowie die journalistische Medienberichterstattung. Und zweitens sind neben den Werkschaffenden sowie der Werke verwertenden Industrie nur scheinbar Prosumer der wahre Kern des Erkenntnisinteresses. Dies macht sich schon darin bemerkbar, dass nicht 
die Internetnutzer/innen mit ihren Bedürfnissen und Standpunkten, sondern ihre Urheberrechtsverletzungen Gegenstand der meisten Studien zum Thema Urheberrecht sind. Wissenschaftler/innen vorinterpretieren urheberrechtliche Bestimmungen als per Gesetz geltende und zwingend zu beachtende Dekrete. Sie projizieren ihre eigene Auffassung auf ein «richtiges» Soll-Verhalten ihrer Zielgruppe und verorten «deviantes» Denken und Handeln als unzulässigen, zu korrigierenden Verstoss. Subjekte, die das Urheberrecht kritisch betrachten und sich nicht rechtskonform verhalten, werden somit zu Tätern/-innen stigmatisiert. Dabei wird vernachlässigt, dass das deutsche Urheberrechtsgesetz ein Konglomerat äusserer Setzungen ist, die keineswegs auf gleichberechtigten Aushandlungsprozessen der gesellschaftlichen Akteure/-innen beruhen, weshalb seine Legitimierung durchaus zu diskutieren wäre.

Es stellt sich die berechtigte Frage: Warum sollte ein nicht rechtskonformes Verständnis von Prosumern entsprechend dem aktuell geltenden Urheberrecht geändert werden müssen, obgleich auch die rechtlichen Bestimmungen an das jeweilige Verständnis der Prosumer angepasst werden könnten? Warum werden nicht alle Akteure/-innen, die vom Urheberrecht potenziell betroffen bzw. daran beteiligt sind, in einen richtungsoffenen Diskurs einbezogen?

\section{Unzureichender Stand der Forschung}

Der bisherige Forschungsstand zum Urheberrecht im Web 2.0 weist zwar eine beträchtliche Anzahl von wissenschaftlichen Publikationen auf, jedoch offenbart sich bei näherer Betrachtung: Weder innerhalb der einzelnen Wissenschaftsdisziplinen noch interdisziplinär kann von einer befriedigenden wissenschaftlich fundierten und zugleich umfassenden Bearbeitung der Thematik gesprochen werden. Obgleich Rechts-, Geistes-, Sozial- und auch Wirtschaftswissenschaften gleichermassen Interesse an wissenschaftlich fundierten Erkenntnissen haben, existieren kaum fächerübergreifende Studien, geschweige denn Untersuchungen, die das menschliche Subjekt und seine Artikulationen in den Mittelpunkt des Erkenntnisinteresses rücken.

Auf (teils un-)verlässlichen Forschungsergebnissen basierende «Handlungsempfehlungen» stammen ganz überwiegend aus der Rechtswissenschaft (z. B. Bauer 2011; Kreutzer 2011; Santangelo 2011). In der Regel wird in den betreffenden Publikationen eine Anpassung einzelner Rechtsnormen an die Digitalisierung der Medien gefordert, obgleich es ihnen an empirischen Grundlagen mangelt. Rechtswissenschaftler/innen sind zwar durchaus darum bemüht, hierbei aus der Sicht ihrer Zielgruppe, den Internetnutzern/-innen, zu argumentieren (z. B. Bauer, 2011). Doch die angewandte Methodik der rechtswissenschaftlichen Analysen lässt im Grunde keine verlässlichen Aussagen über sie zu, da nicht die Menschen und ihre subjektiven Denkund Handlungsmuster untersucht werden, sondern Rechtsnormen und wie man diese nach Ansicht der Autoren/-innen auslegen sollte. 
Auf Empirie beruhende Studien zum Urheberrecht werden von Interessenverbänden der Industrie (z. B. Börsenverein des Deutschen Buchhandels 2010; GfK 2013; Martens et al. 2012) oder durch sonstige wirtschafts- bzw. marktorientierte Auftragsforschung (z. B. Institut für Demoskopie Allensbach 2012; Schwartmann 2012) initiiert. Doch auch die Validität ihrer Forschungsergebnisse gibt zu denken: So erfasst die quantitative empirische DCN-Studie (Studie zur digitalen Content-Nutzung) jährlich als «repräsentativ» deklarierte Daten zum Rechtsbewusstsein - im Sinne eines Bewusstseins für Urheberrechtsverletzungen - und zu Einstellungen zum Urheberrecht (vgl. GfK 2013, 4, 13). Die gewählten Frageformulierungen und Items scheinen die wertgeladenen Sollvorstellungen der industriellen Verbände, die sie in Auftrag gaben, zu spiegeln. Implizit wird davon ausgegangen, dass die von Gesetzeswegen her als illegal geltenden Urheberrechtsverstösse zugleich illegitim seien. Es wird dabei vorausgesetzt, das Urheberrecht müsse von allen Gesellschaftsmitgliedern anerkannt werden. Wie insbesondere junge Menschen die urheberrechtlichen Bestimmungen im Web 2.0 aber tatsächlich auffassen und welche Motive ihr Medienhandeln moderieren, ist für die Forschenden - wenn überhaupt - nur marginal von Interesse.

Einen Nachholbedarf gab es bislang auch in der Medienwissenschaft - und damit in der Medienpädagogik als Teildisziplin, in der das Thema Urheberrecht lange Zeit nur als Randphänomen betrachtet wurde (z. B. Rakebrand et al. 2012; Schemmerling u. Gerlicher 2013). Mit der qualitativen empirischen Untersuchung zum subjektiven Verständnis junger Erwachsener vom Urheberrecht im Web 2.0 (Rakebrand 2014) wurde dem Thema endlich die Aufmerksamkeit gewidmet, die aus sozialwissenschaftlicher Perspektive dringend erforderlich war.

\section{Theoriegeleitete Bezüge und methodisches Vorgehen}

Um Aussagen darüber treffen zu können, wie junge Erwachsene das bestehende Urheberrecht im Zeitalter der zunehmenden Online-Nutzung auffassen, müssen ihre Sichtweisen in das methodische Erhebungskonzept einer empirischen Untersuchung einbezogen werden. Nur durch eine Orientierung an Prosumern ist es legitim, (medienpädagogische oder sonstige) Handlungsempfehlungen zu formulieren. Ihre Artikulationen empirisch zu analysieren und zu interpretieren, bereichert die aktuellen Debatten zum Urheberrecht im Web 2.0 um einen notwendigen Beitrag der Sozialwissenschaft.

Sich an der Zielgruppe zu orientieren, über die fundierte Aussagen getroffen werden sollen, erfordert neben der Auseinandersetzung mit dem Untersuchungsgegenstand (siehe 2) auch das Herstellen entsprechender theoriegeleiteter Bezüge. Im Falle der hiesigen Studie waren dies subjektorientierte Erkenntnisse und Überlegungen (vgl. hierzu Schorb u. Theunert 2000), unter anderem zur Moralentwicklung (Kohlberg 1996), Mediensozialisation (Theunert u. Schorb 2004) sowie Medienaneignung (Schorb 2007) und Medienkompetenz (Schorb 2005). Für Letzteres 
wurde herausgestellt, dass Prosumer in der Lage sein müssen, urheberrechtsrelevante Vorgänge zu identifizieren, zu reflektieren und zu bewerten, um schliesslich urheberrechtsbezogen, das heisst in bewusster Bezugnahme auf das Wissen und die Bewertung von Urheberrecht und urheberrechtlichen Bestimmungen, eine Position beziehen und selbstbestimmt agieren zu können.

Die Forschungsleitenden Fragen wurden durch eine Methodenkombination operationalisiert. Mittels Fragebögen, Gruppendiskussionen und fokussierter leitfadengestützter Intensivinterviews wurde eruiert, was junge erwachsene Internetnutzer/innen über das Urheberrecht wissen, welche persönlichen Erfahrungen sie damit (im Netz) gemacht haben, wie sie es bewerten und welche Beweggründe ihr urheberrechtsbezogenes Internethandeln hat. Im Zentrum der Untersuchung stand damit das subjektive Verständnis von Prosumern bezüglich des Urheberrechts im Web 2.0. Die Kohorte der jungen Erwachsenen im Alter von 18 bis 26 Jahren wurde fokussiert, da sie Web 2.0-Anwendungen - und damit potenziell User Generated Content - vergleichsweise häufig nutzen (vgl. Eimeren u. Frees 2013) und weil sie zudem bereits weitgehend selbstständige Handlungsmuster, ein Werte- und Normsystem sowie ein ethisches, moralisches und politisches Bewusstsein entwickelt haben (vgl. Hurrelmann 2010, 26ff., 33ff., 37f.)

Die digitalen Fragebögen wurden allen Rekrutierten via E-Mail zugeschickt. Sie hatten zum einen den Zweck, Kontextinformationen über die Untersuchten hinsichtlich des Erkenntnisinteresses der Studie in Erfahrung zu bringen, um diese später in die Auswertung einzubeziehen. Zum anderen wurden die Teilnehmer/innen auf Basis ihrer Angaben in zwei Diskussionsgruppen eingeteilt. Sie unterschieden sich dahingehend, dass Urheberrecht bei keinem Mitglied der einen Gruppe (fünf Angehörige) ein dezidierter Bestandteil der Ausbildung oder des Studiums war, jedoch weitgehend bei den Mitgliedern der anderen Gruppe (sechs Angehörige). Diese liessen mehr fachliche Expertise und eine tiefer gehende Auseinandersetzung mit den urheberrechtlichen Bestimmungen erkennen.

Im Zentrum der beiden Gruppengespräche standen die Interaktionen der Untersuchten und somit mehrere subjektive Perspektiven zum Thema «Urheberrecht im Web 2.0». Die Diskussionen wurden im Juni 2013 durchgeführt und dauerten jeweils etwa 150 Minuten. Sie wurden per Video aufgezeichnet, verschriftlicht und im Anschluss systematisiert sowie deskribiert. Bei der Auswertung wurden Rekonstruktionen der jeweiligen Diskursorganisationen (vlg. Lamnek 2005, 203-211) vorgenommen sowie Intergruppenvergleiche mit der Methode der komparativen Analyse (vgl. Bohnsack 2000, 383) angestellt.

Mit jeweils drei ergiebigen Fällen aus den Diskussionsgruppen wurden im Anschluss fokussierte leitfadengestützte Interviews geführt. Dies hatte nicht nur den Vorteil, dass eventuelle Meinungstendenzen in den Gruppengesprächen durch die Aussagen der nachträglich befragten Einzelfälle relativiert oder bestätigt werden 
konnten. Auch war es möglich, sich als Forscher/in rückzuversichern, ob die interviewte Person oder die gesamte Gruppe als solche in der vorherigen Diskussion richtig verstanden worden war. Zudem konnten bisher nicht oder nur oberflächlich angesprochene Aspekte des Erkenntnisinteresses erfragt werden, etwa das individuelle Wissen zum Urheberrecht oder Begründungen der Subjekte, die in den Gruppendiskussionen nicht adäquat erhoben werden konnten. Die sechs Einzelgespräche wurden im Zeitraum von Mitte Juni bis Anfang Juli 2013 durchgeführt und dauerten zwischen 35 und 200 Minuten. Sie wurden via Audio aufgezeichnet und verschriftlicht. Wie bei den Gruppendiskussionen wurden die Transkripte systematisiert und deskribiert. Aus der anschliessenden Formulierung der Interpretationsspuren resultierten sechs Einzelfallinterpretationen, in die auch die Aussagen der jeweiligen Individuen aus den Gruppengesprächen sowie ihre Angaben in den Fragebögen einbezogen wurden (siehe auch Schorb u. Theunert 2000).

\section{Ergebnisse der Untersuchung}

In Hinblick auf den Aussagewert der Untersuchungsergebnisse sei konstatiert, dass lediglich Erkenntnisse über die Altersgruppe der jungen Erwachsenen in Bezug auf das deutsche Urheberrecht in Zusammenhang mit User Generated Content getroffen werden können. Zudem wurden mit dem gewählten Forschungsdesign Einzelfälle untersucht, sodass zwar keine allgemeingültigen Aussagen über die «typischen» Prosumer getroffen werden können. Jedoch sind die gewonnenen Erkenntnisse eine bis dato einmalige Momentaufnahme, die Hinweise auf das subjektive Verständnis des urheberrechtsrelevanten Medienhandelns im Web 2.0 liefern und somit die aktuellen urheberrechtsbezogenen Debatten um einen dringend erforderlichen Beitrag der qualitativen Sozialforschung bereichern.

\section{Junge Erwachsene wollen das Urheberrecht verstehen, doch durchdringen es nicht}

Welches Verständnis haben junge Erwachsene vom Urheberrecht und seinen Bestimmungen im Zusammenhang mit User Generated Content?

Dieforschungsleitende Frage impliziert, junge Erwachsene Prosumer besässen bereits ein Verständnis vom Urheberrecht im Web 2.0, das es zu eruieren gilt. Tatsächlich aber ist es der erklärte Wunsch der Untersuchten, ein solches Verständnis zunächst zu erlangen, respektive die urheberrechtlichen Bestimmungen und ihre Tragweite für das eigene Online-Handeln zu verstehen. So wird gefragt: «Gehört das dann der Welt oder YouTube?» (Cosima). Hat man sein Urheberrecht freiwillig verwirkt, wenn das eigene Werk erst einmal ins Internet gelangt ist? Und teilt man es dann mit der gesamten globalen Gesellschaft oder «nur» mit der OnlineIndustrie? 
Ein Verständnis zu entwickeln bedeutet für die Untersuchten, die Fähigkeit zu erringen, das Urheberrecht auf Basis von Wissen nachvollziehen und beurteilen zu können. Dafür ist nötig, sich entsprechende Informationen anzueignen, die nach ihrer Auffassung aber nicht moralisch aufgeladen sein dürfen. Ein Problem stellt für die jungen Erwachsenen dar, dass die urheberrechtsbezogenen Regelungen zu komplex sind beziehungsweise als zu komplex wahrgenommen werden, um sie durchdringen zu können. Dazu trägt auch negativ bei, dass die urheberrechtsbezogenen Handlungsregeln im Internet als unklar, unübersichtlich, nicht transparent, restriktiv und rückständig aufgefasst werden. Hinzu kommt, dass die Prosumer zum Thema Urheberrecht kaum einen Lebenswelt- beziehungsweise Alltagsbezug herstellen. In der Folge erwerben sie kein vollständiges Wissen über urheberrechtliche Bestimmungen und urheberrechtsrelevante Vorgänge im Web 2.0, können sie daher auch nicht richtig einordnen und demnach nicht souverän im Internet agieren.

Das Urheberrecht soll primär geistiges Eigentum der Urheber/innen schützen, nicht aber das finanzielle Auskommen Dritter sichern

Obgleich sie das Urheberrecht nicht vollends erfassen können, nehmen die Untersuchten Bewertungen vor. Die beiden durchgeführten Diskussionen offenbaren, dass diesbezüglich nur partiell ein Gruppenkonsens erzielt wird. Die einzelnen Subjekte sind der Thematik gegenüber weder eindeutig positiv noch eindeutig negativ eingestellt. Grundlegend jedoch wird die Idee des Urheberrechts im Web 2.0 begrüsst.

Seinen Sinn sehen die Untersuchten fallübergreifend im Schutz des geistigen Eigentums von Urhebern/-innen. Als Künstler/in in fairem Masse Geld zum eigenen Auskommen zu verdienen, wird von einem Grossteil der Untersuchten als notwendig erachtet. Jedoch wird dies im Vergleich zur Anerkennung als Schöpfer/in und zum Schutz vor geistigem Diebstahl nur als ein Randaspekt angesehen. Eine rein wirtschaftlich motivierte Verwertung schutzfähiger Werke durch Dritte verorten die Prosumer als Instrumentalisierung der Schutzfunktion des Urheberrechts, vor allem, wenn dabei «Lücken» im Recht sowie die Unsicherheit und das Unwissen der Internetnutzer/innen ausgenutzt werden. Abgelehnt wird insbesondere das Profitstreben der Industrie und der juristischen Abmahnagenturen: «Steckt dahinter jetzt wirklich so'n berechtigtes Interesse des Künstlers [...] oder ist es nicht nur, ja irgendwie so'n Streben, dass man irgend'ne Lücke ausnutzt und dann merkt, oh das ist noch so'ne Grauzone. Da könnte man jetzt einfach vielleicht mal mit so'ner Abmahnung reinspringen und da mal schön noch ein paar Tausend Euro rausziehen» (Hagen). 


\section{Das Verständnis junger Erwachsener vom Urheberrecht ist von ihrem subjekti-} ven Zugang geprägt

Das Verständnis der Untersuchten vom Urheberrecht im Web 2.0 ist dahingehend geprägt, welchen subjektiven urheberrechtsbezogenen Zugang sie zu der Thematik haben. Dabei ist es nie nur ein Zugang, den junge Erwachsene aufweisen. Es zeigt sich unter anderem, dass vor allem Eltern, Geschwister und auch Bekannte und deren Erfahrungen entscheidenden Einfluss auf die persönliche Auffassung bezüglich der Thematik haben können. Ebenso einflussreich ist, welchem Beruf oder welcher Freizeitbeschäftigung junge Erwachsene nachgehen. Exemplarisch - wenngleich dies verknappt ist - werden hier zwei Perspektiven nachgezeichnet: die der Prosumer und die der Urheber/innen.

Aus Sicht der Prosumer konstatieren die Untersuchten, der Bezug fremder nutzergenerierter Inhalte im Netz sei unumgänglich. Obgleich nicht intendiert, komme es beim Online-Handeln zwangsläufig auch zu Urheberrechtsverletzungen, die man selbst zu verantworten habe. Im Zeitalter technischer und sozialer Globalität müsse das Urheberrecht dringend reformiert werden, da es zum einen Prosumer verunsichere und kriminalisiere, zum anderen wird unterstellt, die konkrete Ausgestaltung des Urheberrechts werde durch die Dekrete Dritter geprägt, nicht aber durch die Prosumer selbst: «Das Problem ist dann, wer klagt denn [...] zum Thema Urheberrecht? Nur diejenigen, die auch das Geld dafür haben. [...] Da entstehen Entscheidungen für Urheberrecht auf einer Ebene, [...] für die alle ein bisschen weiter unten sind, die, ich sag mal, aus der normalen gesellschaftlichen Ebene» (Jan). Unter den Untersuchten waren auch Urheber/innen. Für sie, die ihre nutzergenerierten Inhalte im Web 2.0 verbreiten, stehen der Schutz der eigenen Werke und die Anerkennung der Urheberschaft deutlich im Vordergrund. Als ambivalent stellt sich bei den Untersuchten die Auffassung dar, statt der Internetnutzer/innen trügen die Urheber/innen selbst Verantwortung dafür, ihre Werke im Internet zu veröffentlichen und damit Urheberrechtsverletzungen zu evozieren. Dass sich die jungen Erwachsenen als Rechteinhaber/innen, respektive als Urheber/innen eine grosse Eigenverantwortung zubilligen, wird schon dadurch deutlich, dass sie zum Schutz der eigenen Werke im Netz technische Massnahmen ergreifen - wie die Verringerung der Auflösung eigener Fotos: «Wenn ich fotografieren gehe, dann sind die Fotos letzten Endes auf dreieinhalbtausend mal zweieinhalbtausend Pixelgrösse. [...] Und wenn ich das bei Facebook hochladen würde, würde das Originalbild bei Facebook liegen. [...] Um [...] das erst mal zu sperren, mach ich die um die Hälfte kleiner» (Adrian).

Junge Erwachsene wünschen sich eine Reformierung des Urheberrechts Die untersuchten jungen Erwachsenen fordern übereinstimmend, das Urheberrecht in seiner jetzigen Ausgestaltung zu reformieren. Die Denkrichtungen der Subjekte sind diesbezüglich jedoch ambivalent: Einerseits besteht der Wunsch 
nach mehr Liberalität im Web 2.0, andererseits nach einem starken Schutz der Werke von Künstlern/-innen im Internet. Einigkeit unter den Untersuchten besteht dahingehend, dass das Internet ihrer Auffassung nach keinen rechtsfreien Raum darstellen dürfe, insofern die Normen des Urheberrechts jedenfalls als legitim empfunden werden.

Legitimität ist ergo ein Kriterium, dem das Urheberrecht nach einer Reformierung gerecht werden müsste. Darüber hinaus jedoch sehen sich die Untersuchten nicht im Stande, eine umfassende und für sie selbst zufriedenstellende Lösung zur Änderung der jetzigen urheberrechtlichen Bestimmungen anzubieten. Zumindest aber können sie relevante Aspekte benennen, die eine Reformierung beinhalten sollte. Von den meisten werden ein moderates Umdenken und eine Anpassung der schon bestehenden Rechtsnormen an die Lebensumstände der Prosumer gefordert. Dass dieser Wandel (siehe 2) bereits erfolge, wenn auch nur behäbig, wird von einem Grossteil der Subjekte begrüsst. Kritisiert wird jedoch, dass in den öffentlichen Debatten um Urheberrecht - die diesen stetigen Anpassungsprozess vorantreiben - die Sichtweisen von Internetnutzern/-innen vernachlässigt würden. Kämen diese dennoch zu Wort, seien sie nach vorherrschender Meinung der Untersuchten nicht in der Lage, sich klar zu positionieren.

Dies ist unter anderem mit ihrem mangelnden Rechtsbewusstsein und der fehlenden gezielten Auseinandersetzung mit der Thematik zu begründen, respektive damit, dass sie das Urheberrecht im Web 2.0 aufgrund seiner Komplexität nicht durchdringen können. Es liegt aber auch daran, dass die Untersuchten die Ausmasse der Debatten nicht kennen beziehungsweise diese nicht aktiv verfolgen. Stattdessen orientierten sie sich an einzelnen, vor allem medialen Informationsgebern/-innen und deren transportierten Standpunkten. Als problematisch daran wird gesehen, dass professionelle Medienmacher/innen nicht vermögen, das komplexe Thema Urheberrecht gänzlich unpopulistisch, differenziert und gleichsam leicht verständlich für Prosumer abzubilden.

Die Untersuchten plädieren daher für eine Vereinfachung und mehr Klarheit der urheberrechtlichen Regeln im Internet. Zudem fordern sie mehr Transparenz hinsichtlich des Entstehens der Rechtsnormen. Fallübergreifend sprechen sie sich dafür aus, dass Internetnutzer/innen durch die Aneignung von Informationen über das Urheberrecht aufgeklärt werden sollen, damit sie selbstbestimmt, kritisch-reflexiv und somit kompetent agieren können. Zum einen wünschen sich die Untersuchten dafür einen Handlungsleitfaden zur Orientierung sowie zur Reduzierung der wahrgenommenen Komplexität des Urheberrechts im Web 2.0. Zum zweiten sind es direkte soziale Bezugspersonen, die als Ansprechpartner/innen gewünscht werden. Warnhinweise, wie Schwartmann (2012) sie empfiehlt, werden von den meisten der Untersuchten abgelehnt, jedenfalls wenn diese wertgeladen sind und an die Moral der Prosumer appellieren sollen. Die jungen Erwachsenen bezwei- 
feln, dass durch ein solches Mittel ein Vertrauen zum Urheberrecht geschaffen werden könne.

\section{Ausblick}

Basierend auf den zuvor dargestellten Ergebnissen lassen sich Desiderate in der Forschung sowie Problematiken hinsichtlich der Führung urheberrechtsbezogener Debatten feststellen. Es werden Handlungsempfehlungen für die (vor allem medienpädagogische) Praxis sowie Erfordernisse an die Industrie und Politik postuliert, um jungen Erwachsenen eine Auseinandersetzung mit der Thematik unter Berücksichtigung ihrer eigenen moralischen Vorstellungen, Interessen und Bedürfnisse zu ermöglichen.

\section{Urheberrechtliche Bestimmungen müssen mit subjektivem Verständnis vom Urheberrecht einhergehen}

Junge Erwachsene haben differenzierte, teils konträre, doch tendenziell positive Haltungen zum Urheberrecht, kritisieren aber übereinstimmend den Status Quo und plädieren für eine Reformierung des als rückständig empfundenen Urheberrechtsgesetzes. Jedoch wird ihr subjektives Verständnis vom Urheberrecht im Web 2.0 ihrer Ansicht nach in den öffentlichen Debatten nicht berücksichtigt. Sie fühlen sich vom Prozess des Zustandekommens und des diskursiven Aushandelns der urheberrechtlichen Bestimmungen ausgeschlossen. Es mangelt zudem an einem unvoreingenommenen Blick darauf, wie sie das Urheberecht sehen. Dies betrifft nicht nur die Forschung, von der beim Thema Urheberrecht zumeist Verletzungen der Rechtsnormen betrachtet und Subjekte als Täter/innen stigmatisiert werden, sondern vor allem die Gesetzgebung und Rechtsprechung sowie die Werke verwertende Industrie, die den grössten Einfluss auf das Urheberrechtsgesetz haben. Es muss allgemein hin davon abgerückt werden, das Verständnis von Prosumern müsse zwangsläufig dem geltenden Urheberrecht angepasst werden, obgleich sich die Rechtsnormen aus dem jeweiligen Verständnis der gesellschaftlichen Akteure/-innen und damit aus einer Vielzahl moralischer Urteile konstituieren müssten. Letzteres fordern die untersuchten jungen Erwachsenen.

\section{Junge Erwachsene brauchen Klarheit und Orientierung, um an urheberrechts- bezogenen Diskursen zu partizipieren}

Wie aber soll von den jungen Erwachsenen festgestellt werden, was konkret am Urheberrecht verändert werden muss, wenn sie nicht im Stande sind, das Gesetz in seiner jetzigen Fassung vollends zu durchdringen und zu bewerten? Und wie sollen sie Änderungswünsche äussern, wenn sie nicht fähig sind, sich argumentativ an den Debatten zu beteiligen?

Einen moralischen Standpunkt zu entwickeln, ist eine wesentliche Voraussetzung, um das Urheberrecht für sich verorten und es überhaupt legitimieren (oder ab- 
lehnen) zu können. Wollen die jungen Erwachsenen tatsächlich über eine Reformierung des Urheberrechts diskutieren, fehlt ihnen die Grundlage: und zwar nicht nur das Vermögen, auf Basis des Rechtsbewusstseins Argumente hervorbringen zu können, sondern auch eine fundierte Position einzunehmen und sich darüber an den Debatten zu beteiligen.

Dies vermag der Schlüssel dafür zu sein, urheberrechtsbezogene Medienkompetenz adäquat fördern zu können - eine Aufgabe, die unter anderem der Medienpädagogik zukommt. Zielvorstellung ist, dass Internetnutzer/innen urheberrechtsrelevante Vorgänge und urheberrechtliche Bestimmungen im Netz erkennen, kritisch reflektieren, darauf bezogen selbstbestimmt und in Eigenverantwortung handeln sowie am urheberrechtsbezogenen Diskurs partizipieren können, um ihre eigenen Vorstellungen kund zu tun und potenziell zu realisieren. Dazu benötigen und wünschen sie ausdrücklich Orientierung von Informations- und Verweisgebern/-innen, und zwar zuvorderst von sozialen als kompetent wahrgenommenen Bezugspersonen.

Medienpädagogik muss für das Urheberrecht sensibilisieren und urheberrechtsbezogene Medienkompetenz fördern

Die berufspraktische Medienpädagogik ist dazu angehalten, Prosumer für das Thema Urheberrecht zu sensibilisieren und ihnen zu ermöglichen, ein eigenes Verständnis entwickeln zu können.

Zum einen muss dafür urheberrechtsbezogenes Wissen vermittelt beziehungsweise dazu anregt werden, sich dieses selbst anzueignen und sich mit dem Urheberrecht aktiv auseinanderzusetzen. Dies könnte - so ein Vorschlag der Untersuchten selbst - durch einen übersichtlichen Handlungsleitfaden geleistet werden. Die aktuellen urheberrechtlichen Bestimmungen könnten mit einem solchen zwar nicht vollumfänglich dargeboten, jedoch Wege der Aneignung von entsprechenden Informationen aufgezeigt werden. Zum anderen müssen Subjekte dazu ermutigt werden, eigene Werturteile zu fällen, statt sich (nur) an der vermeintlichen Mehrheitsmeinung der Internetnutzer/innen, an anderen Moralinstanzen oder auch am Urheberrechtsgesetz selbst zu orientieren.

Für junge Erwachsene ist das Urheberrecht ein komplexes, wertgeladenes, in der Tendenz unattraktives, nicht auf die Lebenswelt bezogenes und nicht alltägliches Thema. Es müssen daher niedrigschwellige, lebensnahe und individuelle Zugänge geschaffen werden - so etwa mittels aktiver Medienarbeit: Da für Prosumer der Schutz und die Anerkennung selbstgenerierter Inhalte zentral sind, könnten - medienpädagogisch angeleitet - eigene Medienprodukte ins Internet gestellt und hierfür Nutzungsrechte selbstbestimmt festgelegt werden. Das abstrakt wahrgenommene Gesetz wird dadurch konkret anwendbar. Die medienpädagogische Arbeit darf jedoch nicht nur die diversen urheberrechtsbezogenen Positionen der 
jeweiligen Zielgruppe, sondern muss auch die des breiten Spektrums gesellschaftlicher Akteure/-innen berücksichtigen. Dieser Appell ist ebenso an die journalistischen Medien gerichtet, die für die Untersuchten neben sozialen Bezugspersonen als Informations- und Verweisgeber/innen von Bedeutung sind.

Darüber hinaus stellen die sogenannten «Warnhinweise» im Internet eine überlegenswerte Option dar. Damit sie von jungen erwachsenen Prosumern in der Breite akzeptiert werden, dürfen sie aber keine moralischen Appelle enthalten. Denn die Prosumer wollen sich ihr eigenes moralisches Urteil bilden. Gänzlich ist abzulehnen, dass sie ausschliesslich durch die zunehmenden repressiven juristischen Konsequenzen von Urheberrechtsverstössen im Internet «sensibilisiert» werden sollen. Stattdessen muss eine prospektive aktive Auseinandersetzung mit der Thematik angeregt werden, damit junge Erwachsene im Netz selbstbestimmt agieren können. Ob ihr autonomes Internethandeln urheberrechtskonform ist oder nicht, ist vor allem dahingehend von Belang, dass sie fähig sind, die möglichen (juristischen) Konsequenzen auf Basis ihres Wissens und ihrer Moralvorstellungen selbst abschätzen zu können. Da Subjekte das Urheberrecht oder einzelne Bestimmungen auch begründet als illegitim auffassen können, ist das bewusste Verletzen von Regeln nicht per se als moralisch verwerflich einzustufen.

Die Sichtweisen von Prosumern fanden bei den Gesetzesnovellierungen der letzten Jahre bis dato keine Beachtung. So obliegt es zum einen der (weiteren) subjektorientierten wissenschaftlichen Forschung, ihnen Gehör zu verschaffen und sie auf diese Weise indirekt am urheberrechtsbezogenen Diskurs teilhaben zu lassen. Zum anderen ist die (medienpädagogische) Praxis dazu angehalten, Menschen zu befähigen, an den urheberrechtsbezogenen Debatten zu partizipieren, indem sie potenzielle Handlungsräume aufzeigt.

\section{Literatur}

Bauer, Christian Alexander. 2011. User Generated Content: Urheberrechtliche Zulässigkeit nutzergenerierter Medieninhalte. Berlin u. Heidelberg: Springer.

Bohnsack, Ralf. 2000. "Gruppendiskussion.»In Qualitative Forschung: Theorie, Methoden, Anwendung in Psychologie und Sozialwissenschaften, hrsg. v. Uwe Flick, 369-384. Reinbek bei Hamburg: Rowohlt.

Börsenverein des Deutschen Buchhandels. 2010. Illegal aber egal? Ein Forschungsüberblick zum Unrechtsbewusstsein von Jugendlichen. Zugriff 9.11.2014. http://www.boersenver ein.de/sixcms/me-dia.php/976/Illegal_aber_egal.pdf.

Bundesgesetzblatt. 2003. "Gesetz zur Regelung des Urheberrechts in der Informationsgesellschaft vom 10.9.2003», Bundesgesetzblatt (BGBI.) 2003 I, 1774. Köln. Bundesanzeiger Verlagsgesellschaft. Zugriff 9.11.2014. http://www.urheberrecht.org/topic/Info-RiLi/ ent/11650.pdf.

Bundesgesetzblatt. 2007. «Zweites Gesetz zur Regelung des Urheberrechts in der Informationsgesellschaft vom 26.10.2007», BGBI. 2007 I, 2513. Köln. Bundesanzeiger Verlagsgesellschaft. Zugriff 9.11.2014. http://www.zbmed.de/fileadmin/user_upload/Ueber_uns/ Verwaltung/bgbl107s2513.pdf. 
Eimeren, Birgit van und Beate Frees. 2013. «Rasanter Anstieg des Internetkonsums. Onliner fast drei Stunden täglich im Netz» Media Perspektiven 7-8/2013, 358-372. Zugriff9.11.2014. http://www.ard-zdf-onlinestudie.de/fileadmin/Onlinestudie/PDF/Eimeren_Frees.pdf.

Gasser, Urs. 2010: «Digitales Neuland: Vertreter der Musik- und Filmindustrie sowie der Buchbranche beklagen den mangelnden Respekt der Internet-Nutzer vor dem Urheberrecht. Derweil entstehen im Netz ganz neue Kulturgüter.» Kulturaustausch: Zeitschrift für internationale Perspektiven, hrsg. v. Institut für Auslandsbeziehungen. Band 60, 76-78. Regensburg: ConBrio.

GfK. 2013. «Studie zur digitalen Content-Nutzung (DCN-Studie) 2013.» Zugriff 9.11.2014. http://t1p.de/gwrg.

Groß, Thomas. 2009. «Soziale Netzwerke und die Transformation des Urheberrechts.»In Soziale Netze in der digitalen Welt: Das Internet zwischen egalitärer Teilhabe und ökonomischer Macht, hrsg. v. Christoph Bieber, 203-213. Interaktiva. Bd. 7. Frankfurt am Main: Campus.

Höffner, Eckard. 2010. Geschichte und Wesen des Urheberrechts. Bd 1. München: Europäische Wirtschaft.

Hurrelmann, Klaus. 2010. Lebensphase Jugend. Eine Einführung in die sozialwissenschaftliche Jugendforschung. 10. Auflage. Weinheim u. München: Juventa.

Institut für Demoskopie Allensbach. 2012. «ALLES KOSTENLOS? Mehrheitliches Verständnis in der Bevölkerung für Schutz des Urheberrechts auch im Internet - ausser in der jüngeren Generation.»Zugriff 9.11.2014. http://www.ifd-allensbach.de/uploads/tx_reportsndocs/prd_1206.pdf.

Kohlberg, Lawrence. 1996. Die Psychologie der Moralentwicklung. Frankfurt am Main: Suhrkamp.

Kreutzer, Till. 2011. «Verbraucherschutz im Urheberrecht. Vorschläge für eine Neuordnung bestimmter Aspekte des geltenden Urheberrechts auf Basis einer Analyse aus verbraucherschutzrechtlicher Sicht.» Verbraucherzentrale Bundesverband e.V. Zugriff 9.11.2014. http://irights.info/userfiles/2011-05-03_Verbraucherschutz_im_Urheberrecht.pdf.

Lamnek, Siegfried. 2005. Gruppendiskussion. Theorie und Praxis. 2. Auflage. Weinheim u. Basel: Beltz.

Lauber-Rönsberg, Anne. 2012. «Raubkopierer und Content-Mafia: Die Debatte um das Urheberrecht.» ApuZ. Aus Politik und Zeitgeschichte. 41-42/2012, 2.10.2012. Bonn. Zugriff 9.11.2014. http://www.bpb.de/apuz/145382/raubkopierer-und-content-mafia-diedebatte-um-das-urheberrecht.

Martens, Dirk, Jan Herfert und Tobias Karbe. 2012. Auswirkungen digitaler Piraterie auf die Ökonomie von Medien: Untersuchung der Effekte von Urheberrechtsverletzung auf die Film-, Musik- und Games-Wirtschaft in Deutschland und der Region Berlin-Brandenburg. House of Research. Institut für Medien- und Marktforschung: Berlin. Zugriff 9.11.2014. www.medienboard.de/WebObjects/Medienboard.woa/media/20587.

Rakebrand, Thomas. 2014. "Gehört das dann der Welt oder YouTube?» - Junge Erwachsene und ihr Verständnis vom deutschen Urheberrecht im Web 2.0. München: kopaed.

Rakebrand, Thomas, Nadine Jünger und Marika Ratthei. 2012. «Der produktiv-gestalterische Umgang Jugendlicher mit Musik.» In Klangraum Internet - Report des Forschungsprojektes Medienkonvergenz Monitoring zur Aneignung konvergenter Hörmedien und hörmedialer Online-Angebote durch Jugendliche zwischen 12 und 19 Jahren, hrsg. v. Bernd Schorb, 38-63. Universität Leipzig. Zugriff 9.11.2014. http://t1p.de/29ks. 
Santangelo, Chiara. 2011. Der urheberrechtliche Schutz digitaler Werke: Eine vergleichende Untersuchung der Schutz- und Sanktionsmassnahmen im deutschen, italienischen und englischen Recht. Schriftenreihe des Max-Planck-Instituts für Ausländisches und Internationales Strafrecht Bd. 125. Berlin: Duncker \& Humblot.

Schemmerling, Mareike und Peter Gerlicher. 2013. «Ergebnisse der aktuellen Teilstudie «ldentitätsarbeit und sozialraumbezogenes Medienhandeln in sozialen Netzwerkdienstenı.» In Teilen, vernetzen, liken: Jugend zwischen Eigensinn und Anpassung im Social Web, hrsg. v. Ulrike Wagner u. Niels Brüggen, 103-140. BLM Schriftenreihe, Bd 101. Baden-Baden: Nomos.

Schorb, Bernd. 2005. "Medienkompetenz.»In Grundbegriffe Medienpädagogik, hrsg. v. Jürgen Hüther u. Bernd Schorb, 257-262. 4., vollst. neu konzipierte Auflage. München: kopaed.

Schorb, Bernd. 2007. «Medienaneignung und kontextuelles Verstehen. Welche Implikate ergeben sich aus dem Konstrukt der Medienaneignung für die Medienforschung?» In Dynamisch-transaktional denken - Theorie und Empirie der Kommunikationswissenschaft, hrsg. v. Werner Wirth, Hans-Jörg Stiehler u. Carsten Wünsch, 252-261. Köln: Herbert von Halem.

Schorb, Bernd und Helga Theunert. 2000. «Kontextuelles Verstehen der Medienaneignung.» In Qualitative Kinder- und Jugendmedienforschung. Theorie und Methoden: ein Arbeitsbuch, hrsg. v. Bernd Schorb u. Ingrid Paus-Haase, 33-57. München: kopaed.

Schwartmann, Rolf. 2012. "Vergleichende Studie über Modelle zur Versendung von Warnhinweisen durch Internet-Zugangsanbieter an Nutzer bei Urheberrechtsverletzungen.» Fachhochschule Köln. Cologne University of Applied Sciences. Kölner Forschungsstelle für Medienrecht. Im Auftrag des Bundesministeriums für Wirtschaft und Technologie. Zugriff 9.11.2014. http://l.hh.de/IFxMNt.

Theunert, Helga und Bernd Schorb. 2004. «Sozialisation mit Medien. Interaktion von Gesellschaft - Medien - Subjekt.»In Jugendsoziologische Sozialisationstheorie: Impulse für die Jugendforschung, hrsg. v. Dagmar Hoffmann u. Hans Merkens, 203-219. Weinheim u. München: Juventa. 